\title{
interview
}

\section{Turbulent transition}

\author{
Clustering of dark and grey solitons in fibre lasers has been found to induce a turbulent regime similar to \\ that of a rapidly flowing fluid in a pipe. Sergei Turitsyn from the University of Aston, UK, explains.
}

Why is it important to study the transition between stable and turbulent operation in a fibre laser?

First, it is significant from a practical viewpoint, to ensure that laser operation remains in a coherent, well-behaved regime during an industrial application or scientific experiment, for example. Understanding the origin of the transition may provide the key for preventing unwanted turbulence. It is also very important from a fundamental viewpoint - for understanding the causes of coherence loss in a physical system. A laser has traditionally been thought of as being a source of pure, coherent radiation. Gaining knowledge regarding how and why a fibre laser's coherence varies when its operational parameters (such as its cavity length or pump power) are changed is very useful. The insights gained may also prove beneficial for other physical systems, such as fluid flow in pipes, which also exhibits turbulent behaviour that starts from stable laminar flow.

\section{What have you learnt from your study?}

The key thing we have learnt is that, in some regimes, laser radiation can become a strange mixture of linear waves and coherent nonlinear structures. The laser radiation might appear to be quite similar on a macroscopic scale in the two regimes (for example, it may have similar spectral profiles in the two regimes), whereas its temporal content could vary greatly on the microscopic level.

What triggers the transition and how do the two regimes differ?

The transition to turbulence can be induced by increasing the cavity length of the laser and/or its pump power. The loss of coherence and the onset of turbulence are believed to be caused by the proliferation and clustering of dark and grey solitons. In the stable, coherent laminar regime, the radiation can be considered to be a one-dimensional condensate formed by a multitude of longitudinal modes - it is spectrally narrow and has an almost constant power. Such a state is similar to stable, laminar flow in a pipe. The second

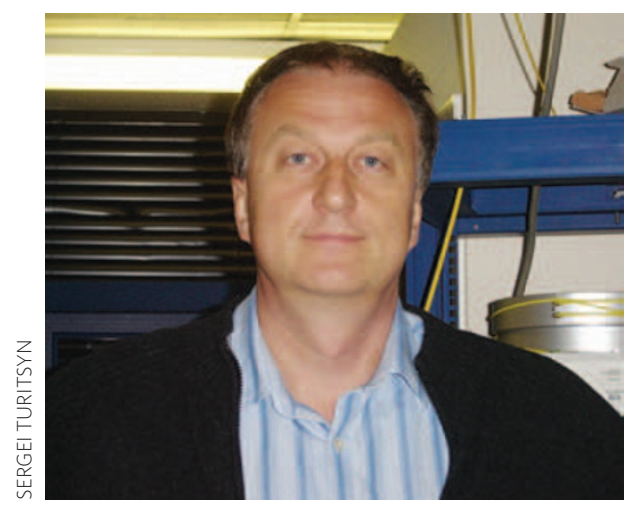

Sergei Turitsyn describes photonics as being an ideal platform for exploring wave turbulence, because it permits a wide range of experiments and measurements to be performed.

regime is one of optical wave turbulence a state far from thermal equilibrium and having many degrees of freedom. A quasicontinuous-wave fibre laser can support millions of laser modes; fluctuations in the amplitude and phase of these modes typically result in stochastic output, which should be described in terms of wave turbulence. I would like to stress that such optical wave turbulence affects very practical properties of laser radiation, such as its spectral shape and thus does have practical relevance.

\section{How will these findings benefit} laser studies?

Understanding the underlying physics and mechanisms of laser operation is certainly important. Operating a device without understanding the physics behind its operation restricts what you can do with it and further developments. Of course, it is possible to use a device without fully understanding how it works, but engineers can always convert knowledge into something useful.

Can you comment on the analogy with hydrodynamics and fluid flow in a pipe?

We discovered that fluid flow in pipes and the propagation of laser light in optical fibres share many things in common. They both lose coherence and become turbulent in fundamentally similar ways - the transition is probabilistic and is triggered by disruption of spatial coherence. We have established the fundamental universality of coherence loss in a linearly stable system. Remarkably, this similarity between fibre laser operation and fluid flow in pipes does not originate from them having similar mathematical descriptions, which is often the case for other known examples of cross-disciplinary analogies. In fluid dynamics, flows are described by the Navier-Stokes equation, whereas our laser is modelled using the modified nonlinear Schrödinger equation; these equations are very different from each other. However, the transition to turbulence is very similar in both cases, indicating that there are some deep connections between the two systems.

\section{What are your plans for future} research in this area?

We now plan to study the characteristics of both optical wave turbulence and stable laser condensate in more detail. I am personally interested in better understanding the coexistence of stochastic radiation and coherent nonlinear structures. At a recent workshop (Advanced Workshop on Nonlinear Photonics, Disorder and Wave Turbulence held in Italy in midJuly), the numerous similarities that exist between turbulence in different fields were discussed. We feel that our research is very timely. Turbulence affects many areas of engineering, including aerodynamics and fluid dynamics, and thus it is critical to gain a good understanding of its behaviour and what causes its onset. Photonics is an ideal arena for investigating the fundamental aspects of wave turbulence because it permits a wide range of controlled experiments and highprecision measurements to be performed. In particular, it allows a large amount of useful information to be rapidly captured.

\section{INTERVIEW BY OLIVER GRAYDON}

Sergei Turitsyn and co-workers have a Letter describing turbulence and coherence loss in a fibre laser on page 783 of this issue. 